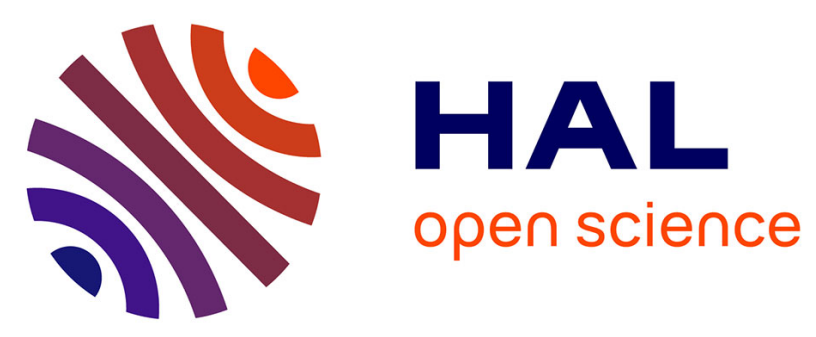

\title{
MRI presentation of hepatocellular carcinoma in non-alcoholic steatohepatitis (NASH)
}

\author{
Fatima Al-Sharhan, Anthony Dohan, Maxime Barat, Adlane Feddal, Benoit \\ Terris, Stanislas Pol, Vincent Mallet, Philippe Soyer
}

\section{> To cite this version:}

Fatima Al-Sharhan, Anthony Dohan, Maxime Barat, Adlane Feddal, Benoit Terris, et al.. MRI presentation of hepatocellular carcinoma in non-alcoholic steatohepatitis (NASH). European Journal of Radiology, 2019, 119, pp.108648 -. 10.1016/j.ejrad.2019.108648 . hal-03487888

\section{HAL Id: hal-03487888 \\ https://hal.science/hal-03487888}

Submitted on 20 Dec 2021

HAL is a multi-disciplinary open access archive for the deposit and dissemination of scientific research documents, whether they are published or not. The documents may come from teaching and research institutions in France or abroad, or from public or private research centers.
L'archive ouverte pluridisciplinaire HAL, est destinée au dépôt et à la diffusion de documents scientifiques de niveau recherche, publiés ou non, émanant des établissements d'enseignement et de recherche français ou étrangers, des laboratoires publics ou privés.

\section{(ㅇ)(1) $\$$}

Distributed under a Creative Commons Attribution - NonCommerciall 4.0 International 


\title{
MRI Presentation of Hepatocellular Carcinoma in Non-Alcoholic SteatoHepatitis (NASH)
}

\author{
Authors \\ Fatima Al-Sharhan, MD¹ (Alsharhan.Fatima@outlook.fr) \\ Anthony Dohan, MD, PhD 1,2 (anthony.dohan@aphp.fr) \\ Maxime Barat, MD1,2 (maxime.baratàaphp.fr) \\ Adlane Feddal, $\mathrm{MD}^{1}$ (adlane.feddal@ gmail.com) \\ Benoit Terris, MD, $\mathrm{PhD}^{2,3}$, (benoit.terris@aphp.fr) \\ Stanislas Pol, MD, PhD ${ }^{2,4}$ (stanislas.pol@aphp.fr) \\ Vincent Mallet, MD, PhD ${ }^{2,4}$ (vincent.mallet@aphp.fr) \\ Philippe Soyer, MD, PhD ${ }^{1,2}$ (philippe.soyer@aphp.fr)
}

\begin{abstract}
Affiliations
${ }^{1}$ Department of Body and Interventional Imaging, Hôpital Cochin, AP-HP, 27 rue du Faubourg Saint-Jacques, 75014 Paris, France

${ }^{2}$ Université de Paris, Paris 5-Descartes, rue de l'Ecole de Médecine, 75006 Paris, France

${ }^{3}$ Department of Pathology, Hôpital Cochin, AP-HP, 27 rue du Faubourg Saint-Jacques, 75014

Paris, France

${ }^{4}$ Department of Hepatology, Hôpital Cochin, AP-HP, 27 rue du Faubourg Saint-Jacques, 75014 Paris, France
\end{abstract}

Conflict of interest statement. All authors declare that they have no conflict of interest to disclose.

Correspondance:

\section{Philippe Soyer, MD, PhD (philippe.soyer@aphp.fr)}

Department of Body \& Interventional Imaging

Hôpital Cochin - APHP, 27 rue du Faubourg Saint-Jacques, 75014 Paris, France

Phone : 33681426781 
Key words: Magnetic resonance imaging (MRI); Hepatocellular carcinoma (HCC); Nonalcoholic steatohepatitis (NASH).

Word count: 2749 words (excluding abstract, references and figure legends)

2 tables, 5 figures

Conflict of interest: The authors have no conflicts of interest to disclose regarding this study.

This research received no financial support.

\section{CRediT author statement}

\begin{tabular}{|l|l|l|l|l|l|l|l|l|}
\hline & FAS & AD & MB & AF & BT & SP & VM & PS \\
\hline $\begin{array}{l}\text { Conceptualization } \\
\text { and Design }\end{array}$ & $\mathbf{X}$ & $\mathbf{X}$ & $\mathbf{X}$ & $\mathbf{X}$ & $\mathbf{X}$ & $\mathbf{X}$ & $\mathbf{X}$ & $\mathbf{X}$ \\
\hline Data curation & $\mathbf{X}$ & $\mathbf{X}$ & $\mathbf{X}$ & $\mathbf{X}$ & & & & X \\
\hline Visualization & $\mathbf{X}$ & $\mathbf{X}$ & $\mathbf{X}$ & $\mathbf{X}$ & $\mathbf{X}$ & $\mathbf{X}$ & $\mathbf{X}$ & $\mathbf{X}$ \\
\hline Resources & & $\mathbf{X}$ & $\mathbf{X}$ & $\mathbf{X}$ & $\mathbf{X}$ & $\mathbf{X}$ & $\mathbf{X}$ & $\mathbf{X}$ \\
\hline Methodology & $\mathbf{X}$ & $\mathbf{X}$ & $\mathbf{X}$ & $\mathbf{X}$ & $\mathbf{X}$ & $\mathbf{X}$ & $\mathbf{X}$ & $\mathbf{X}$ \\
\hline Investigation & & $\mathbf{X}$ & $\mathbf{X}$ & $\mathbf{X}$ & $\mathbf{X}$ & $\mathbf{X}$ & $\mathbf{X}$ & $\mathbf{X}$ \\
\hline Supervision & & $\mathbf{X}$ & $\mathbf{X}$ & $\mathbf{X}$ & $\mathbf{X}$ & $\mathbf{X}$ & $\mathbf{X}$ & $\mathbf{X}$ \\
\hline Validation & $\mathbf{X}$ & $\mathbf{X}$ & $\mathbf{X}$ & $\mathbf{X}$ & $\mathbf{X}$ & $\mathbf{X}$ & $\mathbf{X}$ & $\mathbf{X}$ \\
\hline $\begin{array}{l}\text { Writing- Original } \\
\text { draft preparation }\end{array}$ & $\mathbf{X}$ & $\mathbf{X}$ & $\mathbf{X}$ & $\mathbf{X}$ & $\mathbf{X}$ & $\mathbf{X}$ & $\mathbf{X}$ & $\mathbf{X}$ \\
\hline $\begin{array}{l}\text { Writing - Review } \\
\text { \& Editing }\end{array}$ & $\mathbf{X}$ & $\mathbf{X}$ & $\mathbf{X}$ & $\mathbf{X}$ & $\mathbf{X}$ & $\mathbf{X}$ & $\mathbf{X}$ & $\mathbf{X}$ \\
\hline
\end{tabular}

Fatima Al-Sharhan (FA)

Anthony Dohan (AD)

Maxime Barat (MB)

Adlane Feddal (AF)

Benoit Terris (BT)

Stanislas Pol (SP)

Vincent Mallet (VM)

Philippe Soyer (PS) 


\begin{abstract}
Purpose: To describe the magnetic resonance imaging (MRI) features of hepatocellular carcinoma (HCC) in patients with non-alcoholic steatohepatitis (NASH).

Methods: MRI examinations of 21 patients with HCC and NASH were analyzed by two observers. There were 18 men and 3 women with a mean age of $67.9 \pm 10.2$ (SD) years (range: 36-85 years). Images were qualitatively and quantitatively analyzed with respect to imaging presentation.
\end{abstract}

Results: HCC presented as a single tumor in 13/21 patients (62\%), with a mean longest diameter of $26.9 \pm 20.2$ (SD) $\mathrm{mm}$ (range: $12-88 \mathrm{~mm}$ ); 17/30 HCC (57\%) had a largest diameter $<20 \mathrm{~mm}$. A signal drop between in- and out-of-phase T1-weighted MR images was observed in 16/30 HCC nodules (53 \%). All HCC nodules (30/30; $100 \%$ ) showed hyperenhancement on arterial phase images and 12/30 HCC nodules (40 \%) did not show a wash-out on portal or delayed phase images. Encapsulation was observed in 18/30 tumors (60 $\%)$. MRI findings consistent with liver cirrhosis were present in 16/21 patients (76\%).

Conclusions: Our results show that $57 \%$ of HCC in NASH can present as a lesion smaller than $20 \mathrm{~mm}$ and $40 \%$ do not display wash-out. These results suggest that classical imaging criteria developed for noninvasive diagnosis of HCC should be applied with caution to $\mathrm{HCC}$ in patients with NASH.

Keywords: Hepatocellular carcinoma (HCC); Magnetic resonance imaging (MRI); Malignant tumor; Tissue characterization; Chronic liver disease

\title{
1. INTRODUCTION
}

The imaging features of hepatocellular carcinoma (HCC) in patients with liver cirrhosis have been well-described [1]. The main diagnostic criteria consist of hyperenhancement during the arterial phase and a subsequent wash-out during the portal phase of enhancement on contrastenhanced imaging [2]. When these criteria are both present, the diagnosis of HCC is made with high degrees of confidence, thus obviating the need for further confirmatory tumor biopsy [3]. However, these criteria have been developed for HCCs occurring in patients with liver 
cirrhosis, and not specifically for HCCs that develop in patients with non-alcoholic steatohepatitis (NASH) [4].

Although HCC is more common in the setting of cirrhosis, there is increasing evidence that it can develop in the setting of NASH and that steatosis alone can promote carcinogenesis; of note, one third of NASH-associated HCC occurs in the absence of underlying cirrhosis [5]. In addition, obesity, diabetes, and metabolic syndrome are recognized risks for the development of HCC. The surveillance for HCC in the setting of NASH needs further clarification [5]. European and American guidelines recommend surveillance for HCC with ultrasound on a six-month basis in high risk patients defined by presence of underlying cirrhosis [6]. The incidence of HCC due to NASH is rising. In the same time, patients with NASH are often overweight or obese [7], making a surveillance program with ultrasound difficult. As a consequence, magnetic resonance imaging (MRI) is often performed as a problem-solving tool in patients with NASH to fully exclude or confirm presence of HCC. Whereas, the MRI features of NASH have been reported [8], the MRI features of HCC in patients with NASH have received little attention in the literature so far $[9,10]$

The purpose of this study was to retrospectively analyze the MRI findings of HCC in patients with NASH.

\section{PATIENTS AND METHODS}

\subsection{Patients}

A retrospective search of the database of our institution was performed to retrieve all patients with HCC and NASH who had an imaging examination of the liver in our department of radiology between January 2010 and December 2018. This initial search retrieved a total of 38 patients.

A cross search of the database of the radiology department was then performed to identify those who had MRI of the liver resulting in the exclusion of 17 patients because eight did not have MRI examination, eight had no definite histopathological confirmation of NASH and one had liver tumor that were actually not HCC. A total of 21 patients with a definite diagnosis of HCC and NASH who had undergone MRI examination of the liver were ultimately included and were the study population. There were 18 men and 3 women with a mean age of $67.9 \pm 10.2$ (SD) years (median, 68; range: $36-85$ years). Figure 1 is a flowchart of patients who were considered for this study. 
The diagnosis of HCC was based on the results of histopathological analysis of biopsy specimens obtained from percutaneous biopsy in 19 patients or after surgical resection in two patients. Tissue samples were also obtained from the liver parenchyma to document the status of the underlying hepatic disease using. Fibrosis and cirrhosis were classified as Stage 3 and Stage 4 , respectively $[11,12]$. In all patients, the diagnosis of NASH was originally established by a pathologist with 25 years of experience in liver pathology using the NASH Clinical Research Network criterion, 5 to 10 years before the development of HCC [13]. In addition to histopathological features, NASH was also defined by the absence of HCV-HBV infection, alcohol abuse, and no documentation of primary biliary cirrhosis, primary sclerosing cholangitis, autoimmune hepatitis, hemochromatosis or Wilson disease.

This study received local ethics and institutional review board committee approval. According to the design of this retrospective study, the institutional review board waived the requirement to obtain written informed consent.

\subsection{MRI protocol}

The MR imaging protocol is detailed in Table 1. All patients had undergone abdominal MRI examination using a 1.5-T system (Magnetom Avanto ${ }^{\circledR}$ or Aera ${ }^{\circledR}$, Siemens Healthineers, Erlangen, Germany). The gradient strength of the magnet was $45 \mathrm{mT} / \mathrm{m}$ with a maximal gradient slope of $200 \mathrm{~T} / \mathrm{m} / \mathrm{sec}$. The following sequences were obtained in all patients : free breathing fat-suppressed T2-weighted turbo spin echo (TSE), fat-suppressed breath-hold T2weighted half-Fourier acquisition single-shot turbo spin-echo (HASTE), unenhanced in-phase and out-of- phase T1-weighted imaging, and fat-suppressed three-dimensional volumetric interpolated breath-hold gradient-echo (3D VIBE) sequences before and after intravenous administration of a gadolinium chelate. Examinations were performed with multichannel anterior and posterior phased-array coils. Patients were imaged in supine position. No antispasmodic agents were given to the patients.

In-phase and out-of-phase breath-hold T1-weighted sequences were acquired with a dual echo technique to obtain perfect registration between corresponding images [14].

Breath-hold fat-suppressed T1-weighted 3D VIBE sequences were performed in the transverse plane before and at four times after intravenous administration of gadoterate meglumine (Dotarem ${ }^{\circledR}$, Guerbet, Roissy-Charles de Gaulle, France) at a dose of $0.1-\mathrm{mmol} / \mathrm{kg}$ of body weight, followed by a $20-\mathrm{mL}$ saline flush, using a power injector (Optistar ${ }^{\circledR}$; Mallinckrodt, Cincinnati, $\mathrm{OH}, \mathrm{USA}$ ) at an injection rate of $2-\mathrm{mL} / \mathrm{sec}$. A partial Fourier 
reconstruction (6/8) was used with parallel acquisition (GRAPPA or controlled aliasing in parallel imaging results in higher acceleration factor [CAIPIRINHA]), with an acceleration factor of 2 or 4 . Fat suppression was obtained using spectral selective attenuated inversion recovery (SPAIR) technique. The first acquisition (arterial dominant phase) was obtained 25 sec after the administration of contrast material. The second acquisition (portal dominant phase) was performed $70 \mathrm{sec}$ after the administration of contrast material. The third acquisition was obtained $180 \mathrm{sec}$ after contrast material administration and the final acquisition (equilibrium phase) was performed $5 \mathrm{~min}$ after the start of IV administration of contrast material. All 3D VIBE acquisitions were performed during suspended respiration at end expiration.

\subsection{MR image analysis}

For this retrospective study, two radiologists reviewed the MRI examinations on a picture archiving and communication system (PACS) viewing station (Directview, 12.1.1.1059 version, Carestream Health Inc, Rochester, NY, USA) using a standardized form. To minimize review bias, the radiologists were blinded to any patient information. Agreement was reached by consensus. For all patients qualitative and quantitative variables were tabulated using a standardized data collection form. All MR sequences were assessed as a single set for each individual patient. In patients with more than one HCC nodules, all nodules were analyzed.

HCC nodules were analyzed with respect to tumor long (i.e., largest axial diameter) and short axis (i. e., shortest axial diameter), and absolute and relative (in \%) tumor signal drop between in phase and out of phase T1-weighted MR images. Tumor dimensions were measured using calipers in the axial plane by the two radiologists on magnified images obtained during the portal dominant phase.

Several qualitative variables were evaluated. They included number of lesions (single vs. multiple), tumor location (right vs. left liver), visibility on unenhanced T1-weighted images, visibility on T2-weighted images, tumor hyperenhancement during the arterial phase, wash out of HCC during the portal or delayed phase, tumor encapsulation, endoportal involvement by tumor, presence of visible lymph nodes and involvement of adjacent organs by tumor. Tumor capsule was defined as a peripheral rim of enhancement on portal venous or delayed phase imaging $[15,16]$. Venous and/or portal obstruction by HCC was defined as distention of the hepatic and/or portal vein lumen by an enhancing, endovenous bud originating from the tumor 
[17]. Finally, the two radiologists analyzed hepatic morphology for the presence of MRI features suggestive of liver cirrhosis [18-22].

\section{4. Statistical analysis}

Statistical analysis was performed using software (SAS, version 9.2, SAS Institute, Cary, NC; $\mathrm{R}$, version 2.8, R Foundation, http://www.r-project.org/). Descriptive statistics were calculated for all variables evaluated at MRI. Quantitative (continuous) data were reported as means, standard deviations (SD) and ranges. Qualitative (binary) data were reported as raw numbers, proportions, and percentages.

\section{RESULTS}

At histopathological analysis, 5/21 patients (24\%) had underlying liver fibrosis (S3) and 16/21 patients $(76 \%)$ had liver cirrhosis (S4). A total of $30 \mathrm{HCC}$ nodules were present in the study population, with 21/30 nodules (70\%) in the right liver and 9/30 (30\%) in the left liver. HCC presented as a single tumor in $13 / 21$ patients $(62 \%)$ (Figs. 2, 3) whereas $7 / 21$ patients $(33 \%)$ had two HCC nodules and 1/21 (67\%) three HCC nodules. HCC were located in the left liver only in 5/21 patients (24\%), in the right liver only in 14/21 patients $(67 \%)$ and bilaterally in $2 / 21$ patients $(9 \%)$.

The mean largest diameter of HCC was $26.9 \pm 20.2$ (SD) $\mathrm{mm}$ and the mean shortest diameter was $21.3 \pm 16.4$ (SD) mm (Table 2). Seventeen HCC nodules $(17 / 30 ; 57 \%$ ) had a largest diameter $<20 \mathrm{~mm}$. A signal drop between in- and out-of-phase T1-weighted MR images was observed in $16 / 30$ tumors (53\%), with a mean percentage of $5.6 \pm 8.4$ (SD) $\%$ (range: 0-33 \%) (Fig. 3).

All HCC $(30 / 30 ; 100 \%)$ showed hyperenhancement by comparison to the adjacent hepatic parenchyma on arterial phase images (Figs. 2, 3, 5) and 18/30 HCC (60\%) displayed a wash-out on portal phase and/or delayed phase images (Figs. 2, 5). However, 12/30 HCC nodules $(40 \%)$ did not show a wash-out on portal or delayed images (Fig. 3). All HCC nodules $(30 / 30 ; 100 \%)$ were visible on contrast-enhanced images, $18(18 / 30 ; 60 \%)$ were visible on unenhanced T1-weighed images and 20 (20/30; $67 \%)$ were visible on T2-weighted MR images. Encapsulation was observed in 18 (18/30; 60 \%) HCC nodules (Fig. 5). One HCC nodules $(1 / 30 ; 3 \%)$ was associated with endoportal involvement. No HCC nodules $(0 / 30 ; 0 \%)$ were responsible of involvement of adjacent organs. Findings consistent with liver cirrhosis 
were present in 16/21 patients (76\%). The results of descriptive analysis for qualitative MRI findings are reported in Table 2.

\section{DISCUSSION}

In our study that included 21 patients with HCC and NASH, we found that HCC mostly manifests as a single tumor located in the right liver, with hyperenhancement during the arterial phase after intravenous administration of a gadolinium chelate. Most importantly, we observed that $40 \%$ of HCC nodules in patients with NASH may not display the "wash-out" feature during the portal or delayed phase of enhancement. Hypervascularity during the arterial phase of enhancement and wash-out during the portal phase correspond to the Barcelona criteria for HCC [3]. Therefore, imaging criteria that have been developed for the non-invasive diagnosis of HCC should be applied with caution to HCC in patients with NASH. Another important point is that $57 \%$ of $\mathrm{HCC}$ in NASH manifests as a focal liver lesion smaller than $20 \mathrm{~mm}$.

Ultrasound is frequently the first line imaging modality for surveillance in patients with chronic liver disease at high risk of developing HCC. In patients with NASH, obesity frequently impairs the completeness of ultrasound examination. As reported by del Poggio et al., a body mass index greater than 25 is associated with a failed ultrasound examination of the liver [23] so that computed tomography or MRI are often used to examine the liver. We believe that our study may be helpful as it describes the MRI features of HCC in patients with NASH.

In our study, the mean largest diameter of HCC nodules was $26.9 \pm 20.2$ (SD) mm. This is in line with the results of Yasui et al. who reported a median diameter of $\mathrm{HCC}$ of $3.0 \mathrm{~cm}$ in a series of 87 patients [24]. On the opposite, Iannaconne et al. reported a mean diameter of 8.4 $\mathrm{cm}$ for HCC developed in patients with NAFLD [9]. It may be assumed that the size of the HCC at the time of diagnosis may vary according local practices for surveillance and other variables.

In our study, 13/21 patients (62\%) had HCC presenting as a single mass. Our results are consistent with those of Yasui et al. who reported a single HCC in 65/87 patients (75\%) with NASH [24]. On the opposite, our results are different from those obtained by Iannaccone et al. who reported a single mass in the majority of patients $(21 / 22 ; 96 \%)$ with HCC and nonalcoholic fatty liver disease (NAFLD) [9].

In the present study, all HCC $(30 / 30 ; 100 \%)$ displayed hyperenhancement during the arterial phase after intravenous administration of a gadolinium chelate. In the study by 
Thompson et al. that included 48 patients with NAFLD, arterial phase hyperenhancement was present in 45/48 patients (93\%) [10]. In the study of Elias et al., that included 30 patients with NAFLD, hypervascular nodules were observed in only $2 / 30$ patients $(7 \%)$, consisting in one hepatocellular carcinoma and one high-grade dysplastic nodule [8]. But these authors included patients with NAFLD and not only those with NASH [8].

In our study we found that 12/30 HCC nodules (40\%) in patients with NASH did not display the "wash-out" feature during the portal or delayed phase of enhancement. Of interest, Iannaccone et al. reported a lack of wash-out in only 2/16 HCC (13\%) in patients with NAFLD who underwent MRI [9]. In the study by Thompson et al. that included 48 patients with NAFLD, HCC displayed wash-out in 38/48 patients (79\%) [10].

In our study, a tumor capsule was observed in 18 HCC (18/30; 60 \%) on MRI. Similar results were reported by Iannacconne et al. who reported encapsulation in 15/22 HCC (68 \%) in patients with NAFLD [9]. In the study by Thompson et al. HCC showed encapsulation in 34/48 patients (71\%) with NAFLD [10]. In the study by Rimola et al., in which no patients had NASH, tumor capsule was present in 43/103 HCC (41.7 \%) and was a highly specific feature of HCC (96.5\% specificity) [15]. In another study including 113 non-Asian patients with a variety of underlying chronic liver diseases except NASH, tumor capsule was observed in 63/354 HCC nodules $(17.8 \%)$ [25]. It is admitted that the prevalence of tumor capsule in HCC varies substantially among studies, depending on ethnicity and nature of underlying disease [25-28].

In our study, intratumoral fat was detected in 11/21 HCC (52\%) using a dual echo MR technique. By comparison, in the study of Rimola et al. intralesional fat was detected using MRI in 43/103 HCC (41.7\%) in a population of patients with cirrhosis and no NASH [15]. This supports a similarity in term of fat content between HCC in a general population and those that develop in patients with advanced NASH (i.e., with S3 and S4 NASH) although it has been reported that patients with NASH or NAFLD may have a steatohepatitic HCC [12]. However, steatohepatitic HCC have been reported only in S1 and S2 NASH whereas our patients had either S3 or S4 NASH. [29].

In our study, 5/21 patients (24\%) had underlying liver fibrosis (S3) and 16/21 patients (76\%) had liver cirrhosis (S4). In the study from Japan by Yasui et al. that included 87 patients with NASH, 10 patients (11\%) has S1 NASH, 15 (17\%) had S2 NASH, 18 (21\%) had S3 NASH (i.e., liver fibrosis) and $44(51 \%)$ had S4 NASH (i.e., liver cirrhosis) [24]. In a retrospective analysis of $120 \mathrm{HCC}$ patients NAFLD in the United States, $58.3 \%$ of them had cirrhosis [30]. In our study, MRI findings consistent with liver cirrhosis were present in 16/21 patients $(76 \%)$, who had histopathologically confirmed S4 NASH. In the study by Thompson et 
al. abnormal morphology of the liver suggestive of cirrhosis was present in 22/48 (46\%) patients with NAFLD [10].

Our study has some limitations. Of these, the retrospective nature yielding inclusion bias and the small number of patients are two major limitations. In addition, we did not compare the MRI features of HCC in NASH with those of HCC in patients with virus-induced or alcoholic cirrhosis. Finally, we did not perform a close imaging-pathologic correlation to identify MRI features associated with tumor grade and the impact of tumor fat content on the variation in signal intensity on Dixon imaging. We agree that further prospective studies are needed to determine the actual value of MRI as a screening tool in patients with NASH.

In conclusion, we have described the MRI features of HCC in patients with NASH. Our results suggest that current imaging criteria used for the noninvasive diagnosis of HCC should be applied with caution to HCC in patients with NASH. Further prospective studies are needed to determine the actual value of MRI as a screening tool in patients with NASH. Similarly, more studies are needed to assess the applicability of the LI-RADS criteria to HCC in patients with NASH $[16,31]$.

Conflicts of interest. All the authors listed above had fully participated in the study and approved the publication. All authors have no conflicts of interest regarding this study.

\section{References}

1. Cartier V, Aubé C. Diagnosis of hepatocellular carcinoma. Diagn Interv Imaging 2014;95: 709-719.

2. Cartier V, Aubé C. Gastrointestinal imaging: tips and traps in the diagnosis of small HCC. Diagn Interv Imaging 2013;94: 697-712.

3. McEvoy SH, McCarthy CJ, Lavelle LP, Moran DE, Cantwell CP, Skehan SJ et al. Hepatocellular carcinoma: illustrated guide to systematic radiologic diagnosis and staging according to guidelines of the American Association for the Study of Liver Diseases. Radiographics 2013;33: 1653-1668.

4. Di Martino M, Saba L, Bosco S, Rossi M, Miles KA, Di Miscio R et al. Hepatocellular carcinoma (HCC) in non-cirrhotic liver: clinical, radiological and pathological findings. Eur Radiol 2014;24: 1446-1454.

5. Massoud O, Charlton M. Nonalcoholic fatty liver disease/nonalcoholic steatohepatitis and hepatocellular carcinoma. Clin Liver Dis 2018;22:201-211. 
6. Kolly P, Dufour JF. Surveillance for hepatocellular carcinoma in patients with NASH. Diagnostics (Basel) 2016;6:E22.

7. Yasui K, Hashimoto E, Komorizono Y, Koike K, Arii S, Imai Y et al. Characteristics of patients with nonalcoholic steatohepatitis who develop hepatocellular carcinoma. Clin Gastroenterol Hepatol 2011;9: 428-433.

8. Elias J Jr, Altun E, Zacks S, Armao DM, Woosley JT, Semelka RC. MRI findings in nonalcoholic steatohepatitis: correlation with histopathology and clinical staging. Magn Reson Imaging 2009;27: 976-987.

9 Iannaccone R, Piacentini F, Murakami T, Paradis V, Belghiti J, Hori M et al. Hepatocellular carcinoma in patients with nonalcoholic fatty liver disease: helical CT and MR imaging findings with clinical-pathologic comparison. Radiology 2007;243: 422-430.

10. Thompson SM, Garg I, Ehman EC, Sheedy SP, Bookwalter CA, Carter RE et al. Nonalcoholic fatty liver disease-associated hepatocellular carcinoma: effect of hepatic steatosis on major hepatocellular carcinoma features at MRI. Br J Radiol 2018;91: 20180345.

11. Brunt EM, Janney CG, Di Bisceglie AM, Neuschwander-Tetri BA, Bacon BR. Nonalcoholic steatohepatitis: a proposal for grading and staging the histological lesions. Am $\mathbf{J}$ Gastroenterol 1999; 94: 2467-2474.

12. Takahashi Y, Fukusato T. Histopathology of nonalcoholic fatty liver disease/nonalcoholic steatohepatitis. World J Gastroenterol 2014;20: 15539-15548.

13. Kleiner DE, Brunt EM, Van Natta M, Behling C, Contos MJ, Cummings OW et al. Design and validation of a histological scoring system for nonalcoholic fatty liver disease. Hepatology 2005; 41: 1313-1321.

14. Merkle EM, Nelson RC. Dual gradient-echo in-phase and opposed-phase hepatic MR imaging: a useful tool for evaluating more than fatty infiltration or fatty sparing. Radiographics 2006;26: 1409-1418

15. Rimola J, Forner A, Tremosini S, Reig M, Vilana R, Bianchi L, et al. Non-invasive diagnosis of hepatocellular carcinoma $\leq 2 \mathrm{~cm}$ in cirrhosis: diagnostic accuracy assessing fat, capsule and signal intensity at dynamic MRI. J Hepatol 2012;56: 1317-1323.

16. Ronot M, Fouque O, Esvan M, Lebigot J, Aubé C, Vilgrain V. Comparison of the accuracy of AASLD and LI-RADS criteria for the non-invasive diagnosis of HCC smaller than $3 \mathrm{~cm}$. J Hepatol 2018;68: 715-723.

17. Sandrasegaran K, Tahir B, Nutakki K, Akisik FM, Bodanapally U, Tann M, et al. Usefulness of conventional MRI sequences and diffusion-weighted imaging in differentiating 
malignant from benign portal vein thrombus in cirrhotic patients. AJR Am J Roentgenol 2013;201: 1211-1219.

18. Brancatelli G, Federle MP, Ambrosini R, Lagalla R, Carriero A, Midiri M, et al. Cirrhosis: CT and MR imaging evaluation. Eur J Radiol 2007;61: 57-69.

19. Tonan T, Fujimoto K, Qayyum A. Chronic hepatitis and cirrhosis on MR imaging. Magn Reson Imaging Clin N Am 2010;18: 383-402.

20. Awaya H, Mitchell DG, Kamishima T, Holland G, Ito K, Matsumoto T. Cirrhosis: modified caudate-right lobe ratio. Radiology 2002;224: 769-774.

21. Ito K, Mitchell DG, Gabata T, Hussain SM. Expanded gallbladder fossa: simple MR imaging sign of cirrhosis. Radiology 1999;211: 723-726.

22. Bezerra AS, D'Ippolito G, Faintuch S, Szejnfeld J, Ahmed M. Determination of splenomegaly by CT: is there a place for a single measurement? AJR Am J Roentgenol 2005;184: 1510-1513.

23. Del Poggio P, Olmi S, Ciccarese F, Di Marco M, Rapaccini GL, Benvegnù L et al; Italian Liver Cancer (ITA.LI.CA) Group. Factors that affect efficacy of ultrasound surveillance for early stage hepatocellular carcinoma in patients with cirrhosis. Clin Gastroenterol Hepatol 2014;12: 1927-33.e2.

24. Yasui K, Hashimoto E, Komorizono Y, Koike K, Arii S, Imai Y et al. Characteristics of patients with nonalcoholic steatohepatitis who develop hepatocellular carcinoma. Clin Gastroenterol Hepatol 2011;9: 428-433.

25. Kelekis NL, Semelka RC, Worawattanakul S, de Lange EE, Ascher SM, Ahn IO, et al. Hepatocellular carcinoma in North America: a multiinstitutional study of appearance on T1weighted, T2-weighted, and serial gadolinium-enhanced gradient-echo images. AJR Am J Roentgenol 1998;170: 1005-1013.

26. Stevens WR, Johnson CD, Stephens DH, Batts KP. CT findings in hepatocellular carcinoma: correlation of tumor characteristics with causative factors, tumor size, and histologic tumor grade. Radiology 1994;191: 531-537

27. Freeny PC, Baron RL, Teefey SA. Hepatocellular carcinoma: reduced frequency of typical findings with dynamic contrast-enhanced CT in a non-Asian population. Radiology 1992;182: 143-148.

28. Lafitte M, Laurent V, Soyer P, Ayav A, Balaj C, Petit I, Hossu G. MDCT features of hepatocellular carcinoma (HCC) in non-cirrhotic liver. Diagn Interv Imaging 2016;97: 355360 . 
29. Salomao M, Remotti H, Vaughan R, Siegel AB, Lefkowitch JH, Moreira RK. The steatohepatitic variant of hepatocellular carcinoma and its association with underlying steatohepatitis. Hum Pathol 2012;43: 737-746.

30. Mittal S., Sada Y.H., El-Serag H.B., Kanwal F., Duan Z., Temple S., May S.B., Kramer J.R., Richardson P.A., Davila J.A., et al. Temporal trends of nonalcoholic fatty liver diseaserelated hepatocellular carcinoma in the veteran affairs population. Clin. Gastroenterol. Hepatol. 2015;13: 594.e1-601.e1.

31. Ronot M, Purcell Y, Vilgrain V. Hepatocellular carcinoma: current imaging modalities for diagnosis and prognosis. Dig Dis Sci 2019; 64:934-950.

\section{Legends for figures}

Figure 1. Study flowchart. MRI indicates magnetic resonance imaging. NASH indicates nonalcoholic steatohepatitis. HCC indicates hepatocellular carcinoma.

Figure 2. 69-year-old man with hepatocellular carcinoma at the hepatic dome and nonalcoholic steatohepatitis (F4 cirrhosis).

A, Unenhanced T1-weighted MR image in the axial plane (TR/TE $=6.41 / 2.56 \mathrm{msec})$ shows 13$\mathrm{mm}$, hypointense, focal liver lesion at the hepatic dome (arrow).

B, T2-weighted turbo spin-echo MR image $(\mathrm{TR} / \mathrm{TE}=2000 / 104 \mathrm{msec})$ in the axial plane shows 13-mm hyperintense focal liver lesion at the hepatic dome (arrow).

C, T1-weighted MR image $(\mathrm{TR} / \mathrm{TE}=4.23 / 2.03 \mathrm{msec})$ obtained during the arterial phase following intravenous administration of a gadolinium chelate in the axial plane shows hyperenhancing focal liver lesion at the hepatic dome (arrow).

D, T1-weighted MR image $(\mathrm{TR} / \mathrm{TE}=4.23 / 2.03 \mathrm{msec}$ ) obtained during the portal phase reveals encapsulated focal liver lesion displaying wash-out.

Figure 3. 83-year-old man with hepatocellular carcinoma and nonalcoholic steatohepatitis (F4 cirrhosis).

A, T1-weighted MR image $(\mathrm{TR} / \mathrm{TE}=4.23 / 2.03 \mathrm{msec})$ obtained during the arterial phase following intravenous administration of a gadolinium chelate in the axial plane shows hyperenhancing focal liver lesion in the right liver (arrow). 
B, T1-weighted MR image $(\mathrm{TR} / \mathrm{TE}=4.23 / 2.03 \mathrm{msec})$ obtained during the portal phase reveals no wash-out of the lesion.

C, On T2-weighted turbo spin-echo MR image $(\mathrm{TR} / \mathrm{TE}=2980 / 88 \mathrm{msec})$ in the axial plane the lesion is not visible. Ascites is present (arrow).

Figure 4. 66-year-old woman with hepatocellular carcinoma and nonalcoholic steatohepatitis ( $\mathrm{F} 4$ cirrhosis).

A, In-phase T1-weighted MR image in the axial plane shows large focal liver lesion in the right liver that shows internal spontaneously hyperintense foci (arrow).

B, On out-of-phase T1-weighted MR image, the hyperintense foci become hypointense, indicating fat content (arrow).

Figure 5. 61-year-old man with hepatocellular carcinoma and nonalcoholic steatohepatitis (F3 fibrosis).

A, Unenhanced T1-weighted MR image in the axial plane $(\mathrm{TR} / \mathrm{TE}=4.05 / 1.48 \mathrm{msec}$ ) shows 38 x $26-\mathrm{mm}$, hypointense focal liver lesion in segment 5 of the liver (arrow).

B, T2-weighted HASTE MR image (TR/TE = 1000/90 msec) in the axial plane shows slightly hyperintense focal liver lesion (arrow).

C, T1-weighted MR image $(\mathrm{TR} / \mathrm{TE}=4.05 / 1.48 \mathrm{msec})$ obtained during the arterial phase following intravenous administration of a gadolinium chelate in the axial plane shows hyperenhancing focal liver lesion (arrow).

D, T1-weighted MR image $(\mathrm{TR} / \mathrm{TE}=4.05 / 1.48 \mathrm{msec}$ ) obtained during the portal phase reveals wash-out of the lesion and encapsulation. 


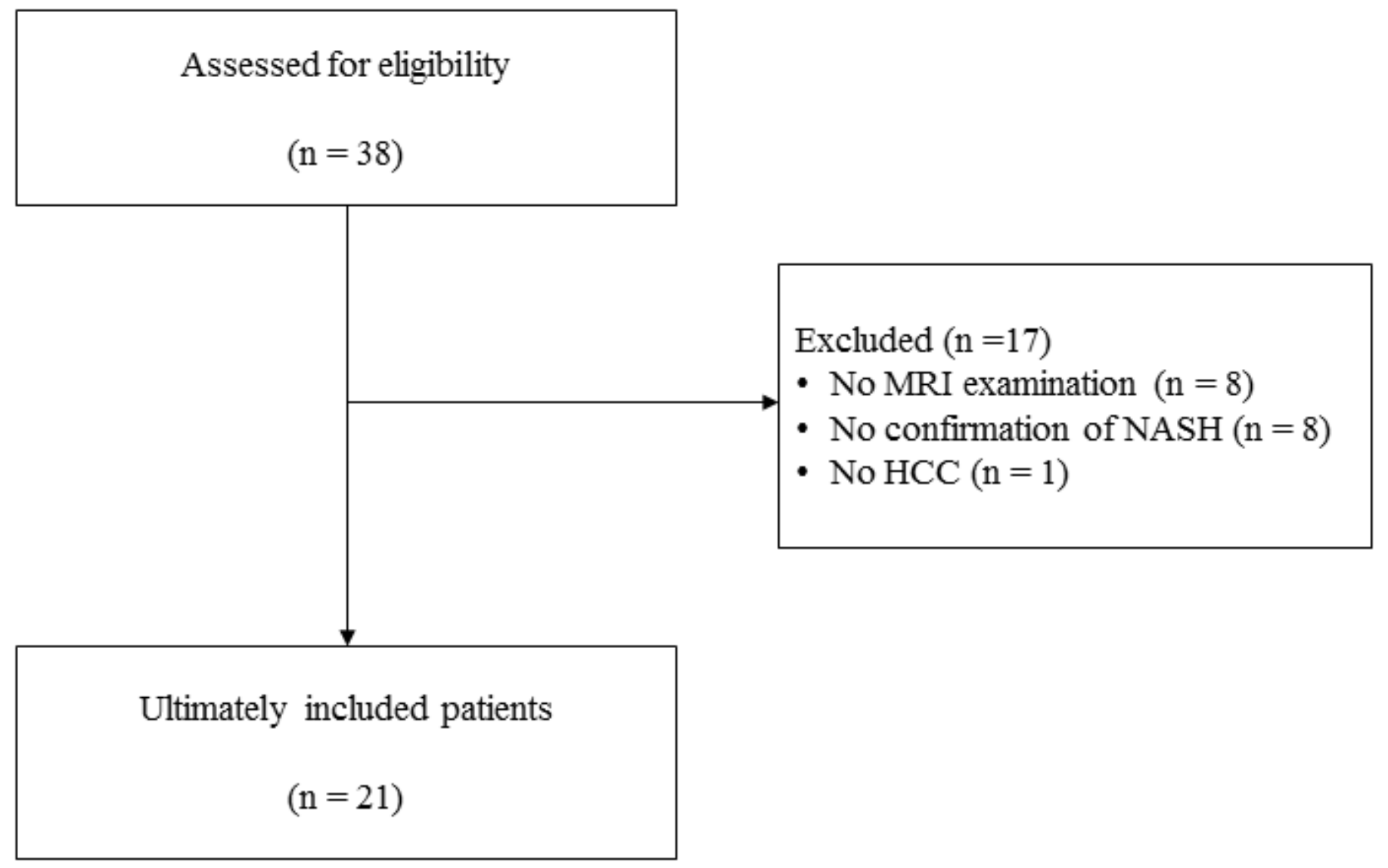





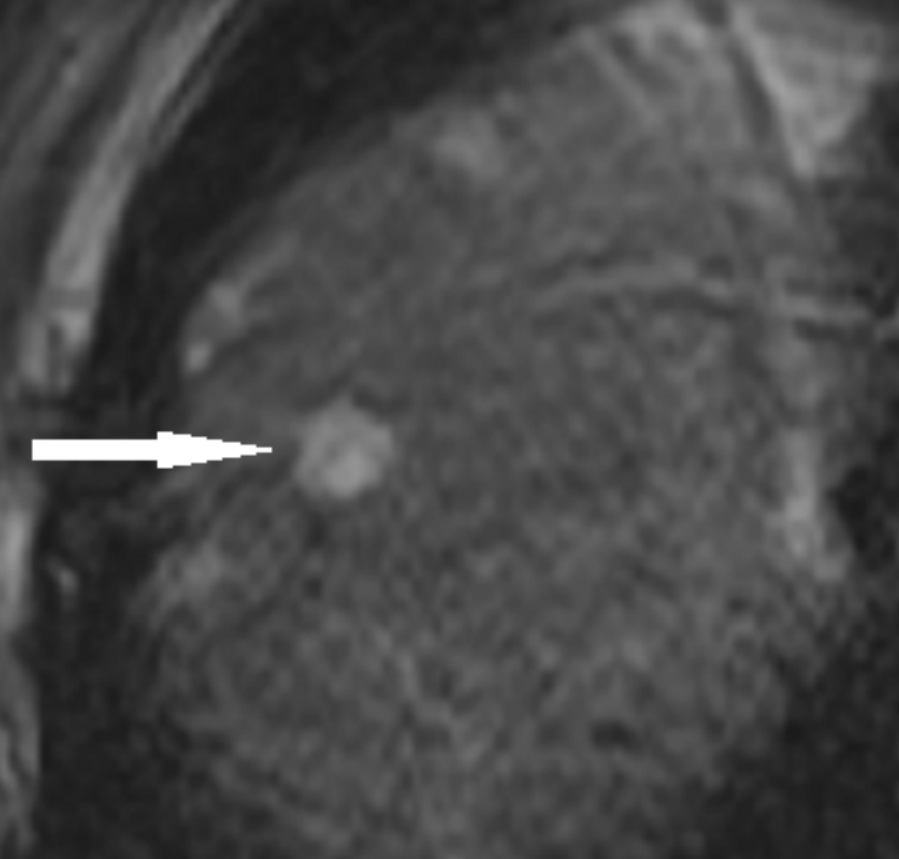





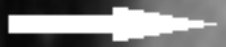




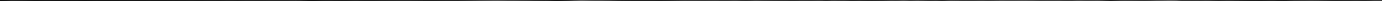




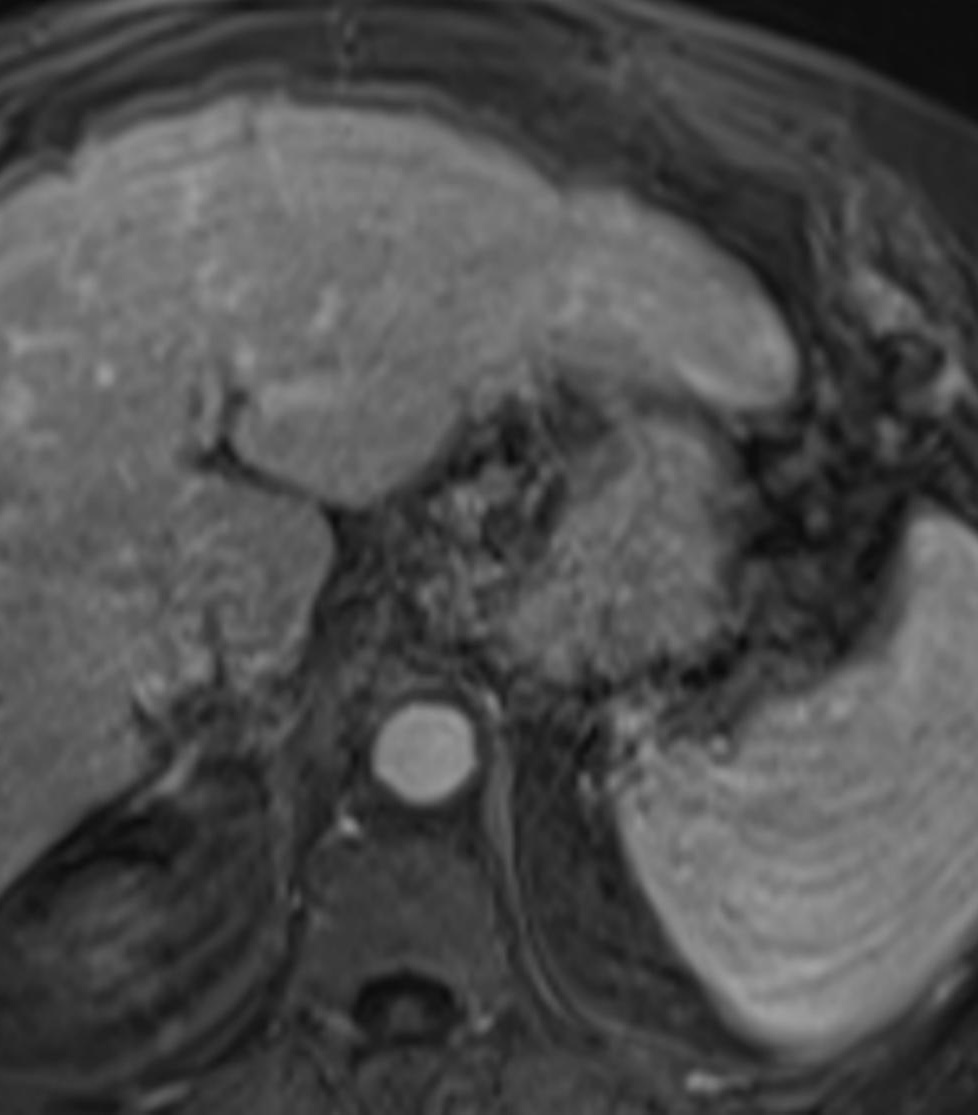









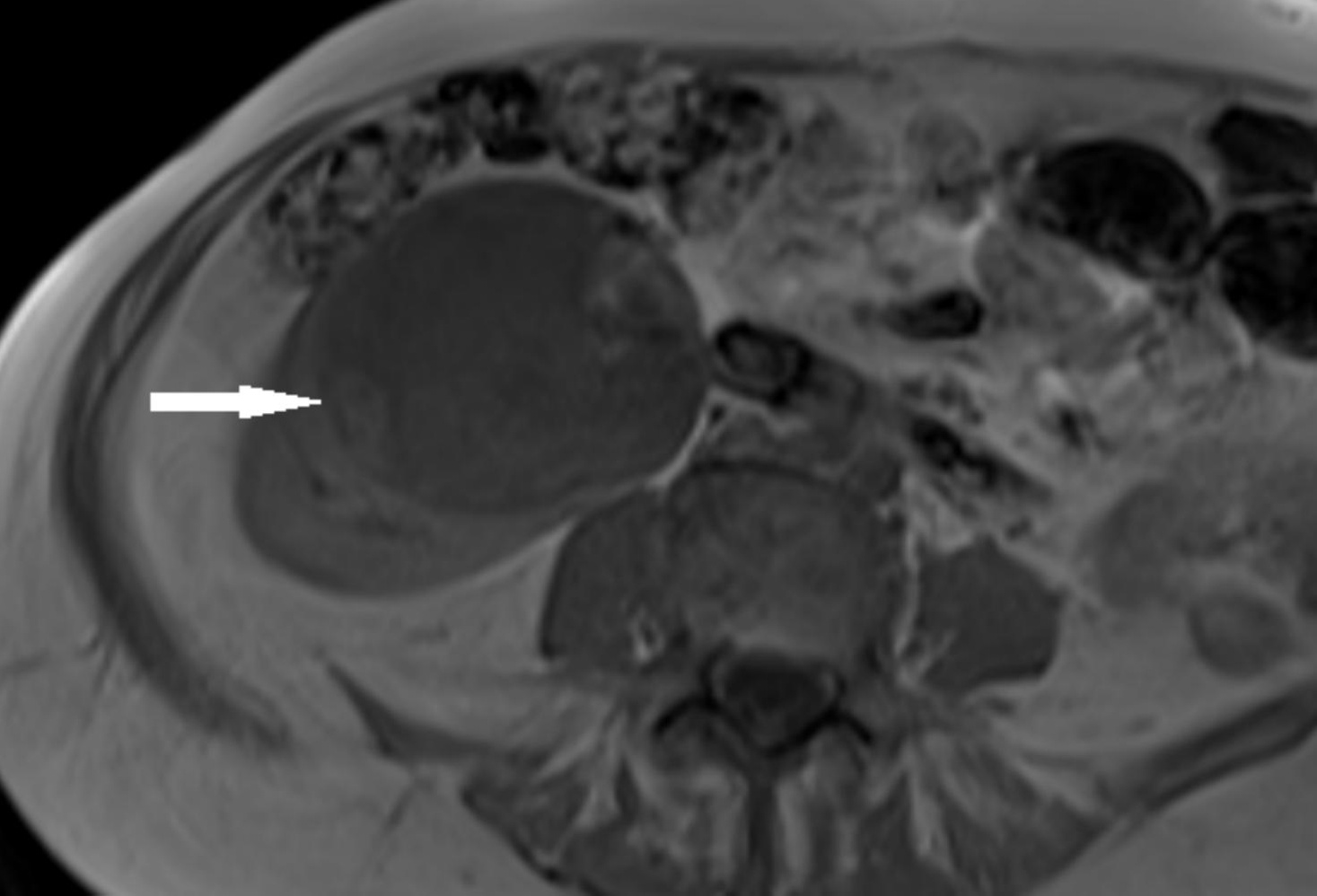





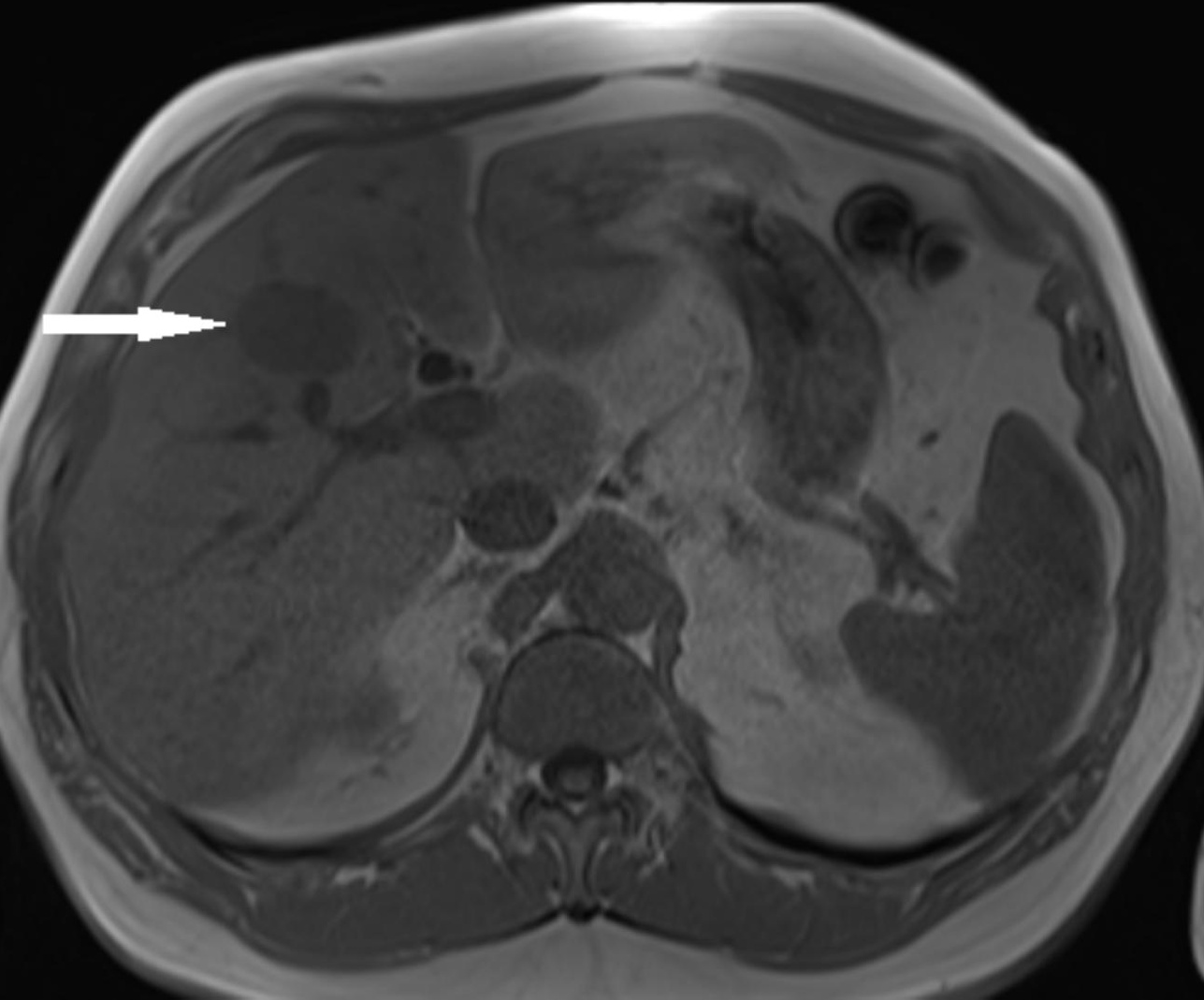




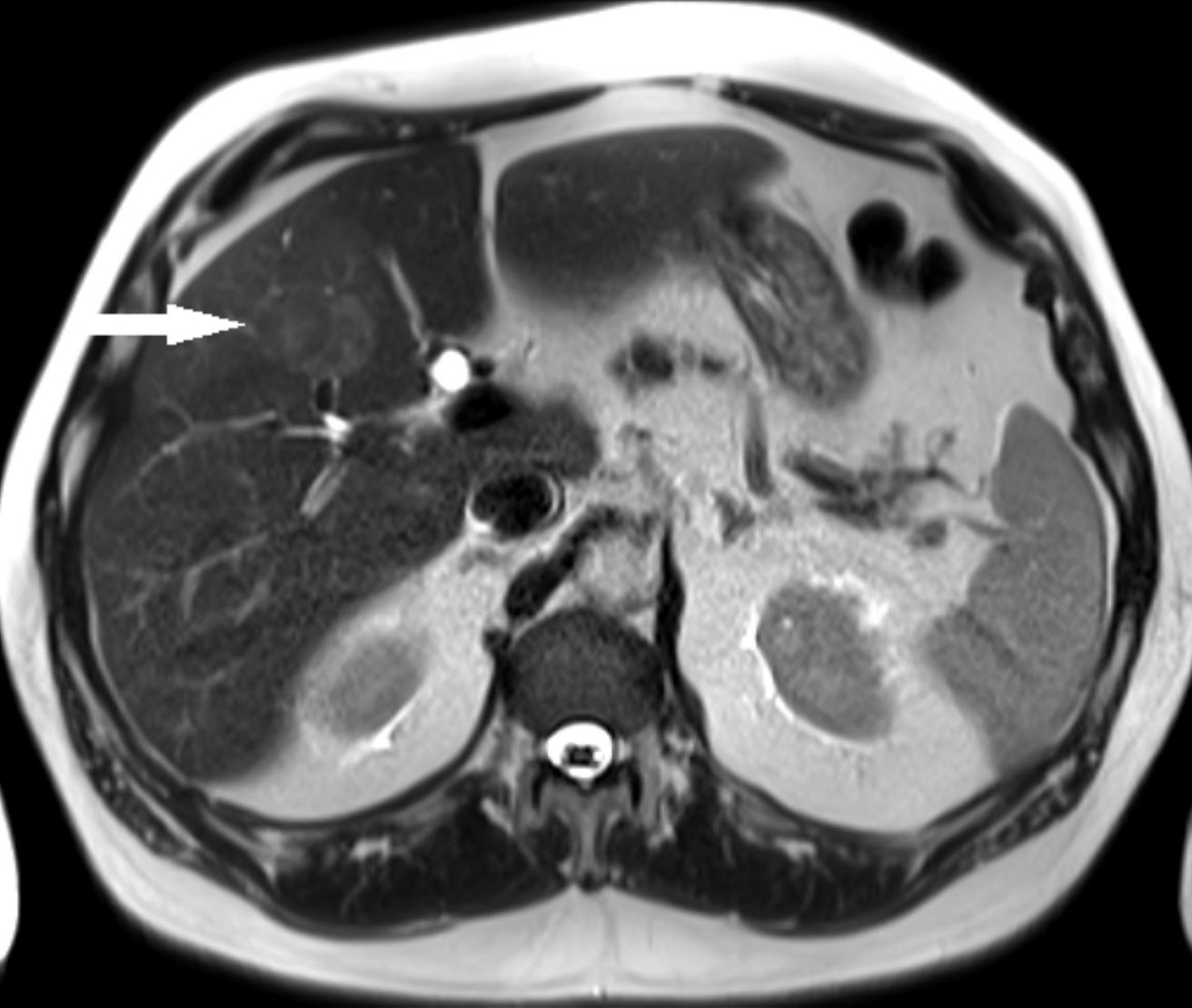




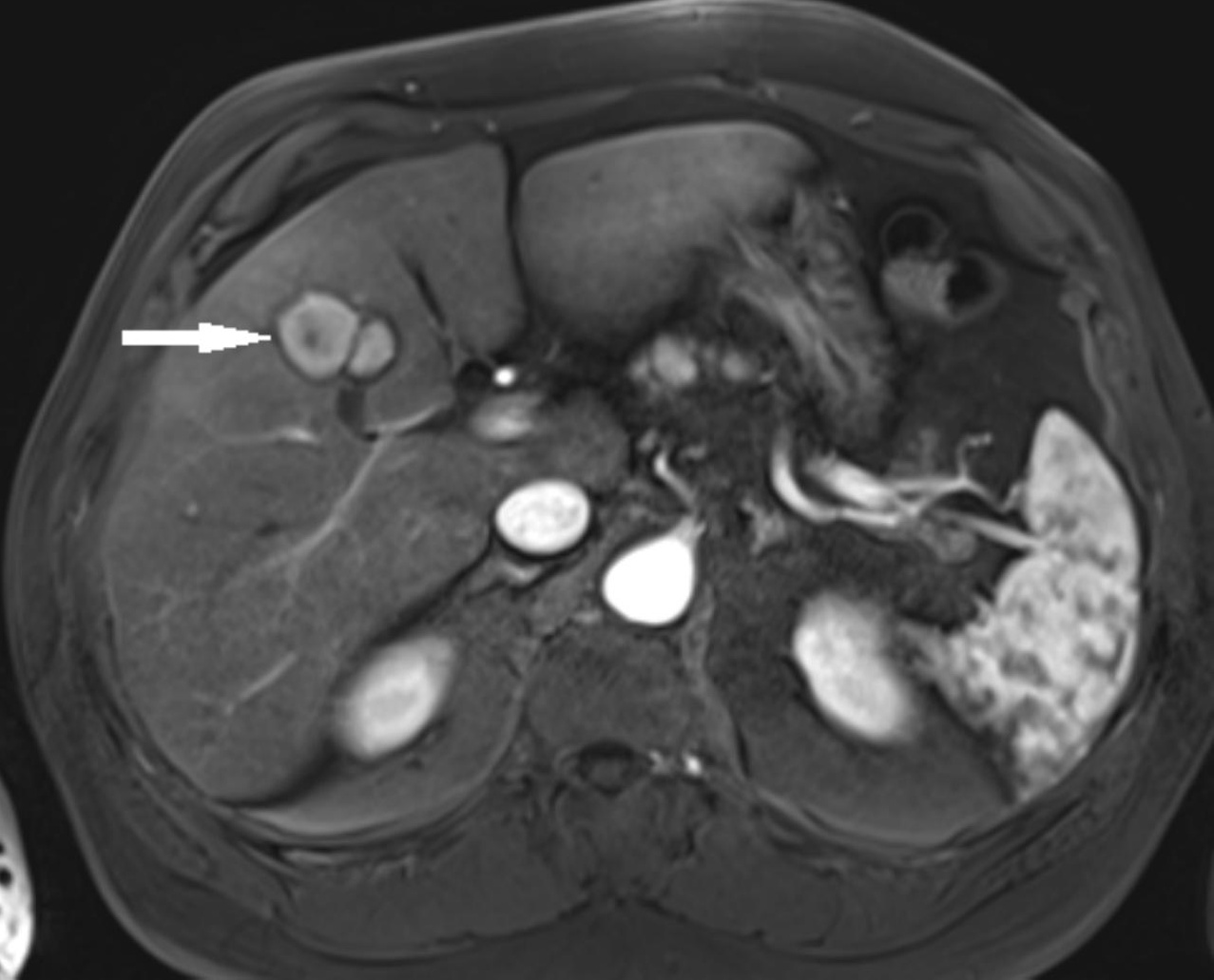




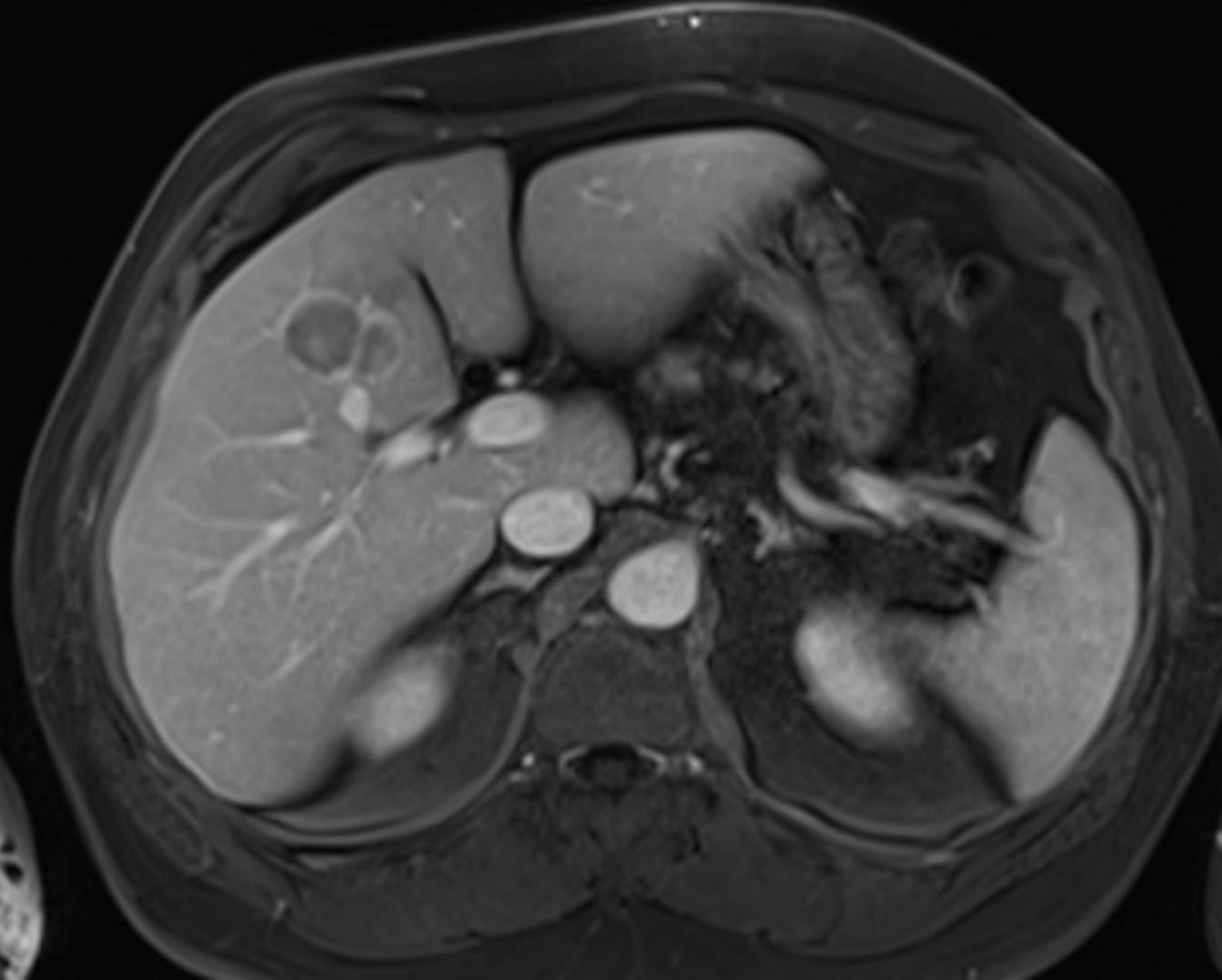


Table 1. MR imaging parameters

\begin{tabular}{|l|l|l|l|l|l|}
\hline Variable & T2 TSE & T1 in phase & T1 out of phase & T2 HASTE & T1 3D VIBE \\
\hline Plane & Transverse & Transverse & Transverse & $\begin{array}{l}\text { Transverse } \\
\text { and coronal }\end{array}$ & Transverse \\
\hline Repetition time $(\mathrm{ms})$ & Variable* & $6.41-110$ & $6.41-110$ & $1000-1200$ & $3.90-4.23$ \\
\hline Echo time $(\mathrm{ms})$ & $88-121$ & $2.56-4.77$ & $1.28-2.39$ & $89-95$ & $1.48-2.03$ \\
\hline Flip angle $\left({ }^{\circ}\right)$ & 180 & $9-10$ & $9-10$ & 150 & $9-14$ \\
\hline Fat suppression & Spectral & No & No & No & Spectral \\
\hline Slice thickness $(\mathrm{mm})$ & $4.5-5$ & $3-7.5$ & $3-7.5$ & $4.5-6$ & 2.2 \\
\hline Intersection gap $(\mathrm{mm})$ & 0 & $0-1$ & $0-1$ & $0-1$ & 0 \\
\hline Number of slices & 24 & $24-80$ & $24-80$ & $19-24$ & $72-120$ \\
\hline Matrix size & $\begin{array}{l}256-320 \\
\mathrm{x} 256-384\end{array}$ & $\begin{array}{l}320-352 \\
\mathrm{x} 182\end{array}$ & $\begin{array}{l}320-352 \\
\mathrm{x} 182\end{array}$ & $256 \times 198$ & $\begin{array}{l}320-384 \\
\mathrm{x} 234-240\end{array}$ \\
\hline Number of signal averages & 1 & 1 & 1 & 1 & 1 \\
\hline Acquisition time $(\mathrm{sec})$ & $150-320$ & $19-28$ & $19-28$ & $24-26$ & $17-23$ \\
\hline
\end{tabular}

Note. TSE indicates turbo spin-echo; HASTE indicates fat-suppressed breath-hold T2-weighted half-Fourier acquisition single-shot turbo spin-echo; 3D VIBE indicates three-dimensional volumetric interpolated breath-hold gradient-echo. ${ }^{*}$ TR depended on the duration of the breath cycle. 
Table 2. Distribution of MRI findings in 30 hepatocellular carcinoma lesions in 21 patients with NASH

\begin{tabular}{|r|l|}
\hline Quantitative variables & \\
\hline Tumor longest axis (mm) & $26.9 \pm 20.2[11-88]$ \\
\hline Tumor shortest axis (mm) & $21.3 \pm 16.4[10-72]$ \\
\hline Tumor signal drop (\%)* & $5.6 \pm 8.4[0-33]$ \\
\hline Qualitative variables & \\
\hline Left liver location & $9(9 / 30 ; 30 \%)$ \\
\hline Arterial phase hyperenhancement & $30(30 / 30 ; 100 \%)$ \\
\hline Tumor fat content & $16(16 / 30 ; 53 \%)$ \\
\hline Visibility on T1-weighted images & $18(18 / 30 ; 60 \%)$ \\
\hline Visibility on T2-weighted images & $20(20 / 30 ; 67 \%)$ \\
\hline Tumor encapsulation & $18(18 / 30 ; 60 \%)$ \\
\hline Endoportal involvement & $1(1 / 30 ; 3 \%)$ \\
\hline Invasion of adjacent organs & $0(0 / 30 ; 0 \%)$ \\
\hline
\end{tabular}

Note. Data are given on a per lesion basis. Quantitative variables are expressed as mean \pm standard deviation; numbers in brackets are ranges. Qualitative variables are expressed as raw numbers; numbers in parentheses are proportions followed by percentages.

$*$ Tumor signal drop between in phase and out of phase imaging was observed in $16 / 30$ HCC lesions and is expressed in \%. Tumor signal drop was null in 14 (14/30; $47 \%$ ) HCC lesions. 